generate deep social consequences that mark human life. Managing a human disaster remains a challenge for the health policy in Brazil. The difficulties faced are related to the lack of interface with other public policies. The urgency to incorporate intervention/action strategies into health plans is important. Implementation of prevention and training programs, and adopting strategies and protocols for the whole network of attention is critical.

Discussion: It is important to emphasize the importance of broadening the theoretical definitions by overcoming the divergences of the concepts adopted between the theoretical and operational field, by elaborating a review of the Brazilian legislation in order to broaden and contemplate the needs of different people.

Prehosp Disaster Med 2019;34(Suppl. 1):s136-s137

doi:10.1017/S1049023X19003005

Human Factor and Disasters: Possible Equations A/Prof. Maria Isabel Barros Bellini, A/Prf. Ines Amaro da Silva, Prof. Beatriz Gershenson, Prof. Michele Cardoso Correa Pontificia Universidade Católica/PUCRS; Escola De Saude Pública/ ESP/SES, Porto Alegre, Brazil

Introduction: This research starts from the assumption that work accidents, in addition to fortuitous or individual phenomena, imply social and organizational factors, and highlights the social character of the production of the accident at work. For this reason, this study investigates the living conditions and the ways of workers in the oil and gas industry in Brazil.

Aim: To analyze the human factors in the relationship with work accidents on oil platforms from the social dimensions.

Methods: It is qualitative research and it has as instruments of collection the focal group and individual interviews with workers and managers of the platforms, participant observation, and documentary analysis.

Results: The research is still being carried out, but some reflections are possible so far: accidents at work depend on the direct or indirect relationship of workers with the work process itself, the modalities of production of work, and management of work. Possible causes underlying the accident are the quality of life and the conceptions of health and safety. Associated with it are social constructs and the multifactorial causes of occupational accidents including the relations between acts and unsafe conditions.

Discussion: The increase in outsourcing and the decrease in training quality, as well as the prioritization of production, targets the detriment of meeting safety criteria. There is a need to reassess labor management, safety policies, and outsourcing processes. Lack of awareness of the proper use of safety equipment and the organization of the work environment are major causes of work-related accidents. The human factor focuses on the individual, group, organizational, and social dimensions in complex interactions. The identification of social processes between working groups in empirical reality, the influence of elements of culture, organizational management, and their impacts on relations and on safe work performance allows an understanding of social risks.

Prehosp Disaster Med 2019;34(Suppl. 1):s137

doi:10.1017/S1049023X19003017

\section{Identification of Preventable Death and Severe Complications in Train Crashes in Rural and Cold Environment Using a Simulation-Based Model} Mr. Jonas Aléx, Mrs. Rebecca Forsberg, Mrs. Heléne Nilsson

Umeå University, Umeå, Sweden

Introduction: The use of rail transport is increasing in Sweden, as well as within Europe, and train speeds are escalating. These factors contribute to an increasing frequency of train crashes and major crashes so severe that they can be classified as disasters. There is a lack of knowledge concerning factors of importance related to the rescue operation that can influence survival rate at train crashes, especially in cold environments.

Aim: The aim was to identify preventable death and severe complications among passengers in a train crash in rural and cold environments using a simulation-based model.

Methods: A train crash scenario was developed based on scientific research, crash reports, and lessons observed in incidents. The scenario was set to a train with seven carriages consisting of 150 passengers that derailed in a curve in $160 \mathrm{~km} / \mathrm{h}, 10 \mathrm{~km}$ from the hospital. In Umeå in the north of Sweden, 12 participants from seven emergency/disaster organizations joined in two preparing workshops and a real-time simulation-based train crash. The Emergo Train System (ETS) was chosen as a simulation tool. Data collection such as rescue capacities, response time, and patient surge were collected and transferred into the ETS. Results: The results show 17 preventable death and 9 preventable severe complications since the actions were not implemented in the recommended time.

Discussion: The results show that an extended rescue operation can have devastating consequences especially in cold environments. Further experimental simulations are needed with defined interventions to find out how preventable deaths and severe complications can be reduced.

Prehosp Disaster Med 2019;34(Suppl. 1):s137

doi:10.1017/S1049023X19003029

\section{Immunization Readiness of a Deploying Emergency Medical Team \\ Ms Melanie Morrow ${ }^{1,2}$, Ms Hollie Sekulich ${ }^{1}$, Ms Abigail Trewin ${ }^{1}$, Dr. Peter Archer ${ }^{1}$ \\ 1. National Critical Care and Trauma Response Centre, Woolner, Australia \\ 2. Royal Darwin Hospital Pharmacy Department, Tiwi, Australia}

Introduction: It is a requirement for a World Health Organization verified Emergency Medical Team (EMT) that all members be immunized against common diseases in the deploying region. Most jurisdictions use private suppliers such as travel doctors for immunization services. When a deployment is announced, members are nominated by their jurisdiction under the condition they are fully immunized. It is up to the individual to monitor their immunization status.

Aim: To determine how many members nominated for deployment were fully immunized.

Methods: Nominated members sent their completed vaccination record to a central location for assessment of their immunization status. The following data were recorded: vaccination 
status, last-minute booster doses required, and the number of emails sent by the assessor in processing the records. The number of phone calls made and received were not recorded.

Results: To complete the skills matrix for a field hospital containing an emergency department and operating theater (an EMT type 2), 61 members were nominated. At the time of assessment, 32 (52\%) were fully immunized, requiring no further booster doses (vaccinations or serology tests). Three members were removed from the deployment as they were not fully immunized. Last-minute booster doses were required by 27 (44\%) members, with a total of 74 booster doses administered (range 0-5). 19 of the booster doses administered were immunizations required to work in any health facility in Australia. The most common vaccines requiring booster doses were rabies $(n=21)$ and typhoid $(n=15) .58$ emails were sent over a period of 5 days to 24 members to clarify vaccination status.

Discussion: This deployment highlighted a gap in members' perception of their immunization status, leading to delays in deployment readiness for the team. A new electronic system where vaccine status tracking occurs in real time should address this issue.

Prehosp Disaster Med 2019;34(Suppl. 1):s137-s138

doi:10.1017/S1049023X19003030

\section{The Impact of the New European Union General Data Protection Regulation (GDPR) on Data Collection at Mass Gatherings \\ Dr. Annelies Scholliers ${ }^{1,2}, \mathrm{Mr}$. Dimitri De Fre ${ }^{2,3}$, Mrs. Inge D'haese ${ }^{2}, M r$. Stefan Gogaert ${ }^{2}$ \\ 1. Department of Anaesthesiology and Perioperative Medicine, University Hospital Brussels, Jette, Belgium \\ 2. Mass Gathering Solutions, Wambeek, Belgium \\ 3. University Hospital Gasthuisberg, Leuven, Belgium}

Introduction: As of May 2018, a new European privacy law called the General Data Protection Regulation (GDPR) is in order. With this law, every organization operating in the European Union (EU), needs to adhere to a strict set of rules concerning collection and processing of personal data.

Aim: To explore the consequences of the GDPR for data collection at mass gatherings in the European Union.

Methods: Since the law was published on April 27, 2016, a thorough reading of the law was conducted by 4 persons with a background in mass gathering health. The GDPR consists of 99 articles organized into 11 chapters. There are also 173 recitals to further explain certain ambiguities. Key articles and recitals relating to healthcare and scientific research were identified. Possible pitfalls and opportunities for data collection and processing at mass gatherings were noted.

Discussion: Under article 4, key definitions are noted. There is a clear definition of "data concerning health". According to the GDPR, health data is a special category of personal data which should not be processed according to article 9(1). However, there is an exception for scientific research (article $9(2)(j))$. There are a few safeguards in place, as laid out in article 89. One interesting point is that according to article $89(2)$, certain derogations can take place if the law interferes with scientific research. The GDPR has major consequences for data collection and processing in the EU. However, with the use of certain safeguards (e.g., pseudonymization) there are still ample opportunities for scientific research. It is important to review one's method of data collection to make sure it complies with the GDPR.

Prehosp Disaster Med 2019;34(Suppl. 1):s138

doi:10.1017/S1049023X19003042

Impact Scale for the Continuity of Care in Contingency Management Situations - Operationalization of the Crisis Standards of Care

Mr. Roel Geene ${ }^{1}$, Dr. Pieter van der Torn ${ }^{2}$, Dr. Dennis den

Hartog ${ }^{3}$

1. Trauma Centre Southwest, Erasmus MC, University Medical Center Rotterdam, Rotterdam, The Netherlands

2. Itineris Consultancy, Rotterdam, The Netherlands

3. Trauma Research Unit, Department of Surgery, Erasmus MC, University Medical Center Rotterdam, Rotterdam, The Netherlands

Introduction: A common language is needed to compare the impacts of incidents, crises, and disasters among health care coalition members, such as emergency medical services, general practitioners, and hospitals. A generic impact scale was developed, based on the Crisis Standards of Care, and was put to the test during the 2017 and 2018 winter/flu-seasons.

Aim: To define an impact scale for the quantitative assessment of the hospital response to incidents, crises, and disasters.

Methods: An impact scale has to be generally applicable to be useful in the context of a health care coalition. It should be applicable to all hazards and all parties in proactive and reactive, real-time settings. In addition, the scale should be easy to understand and score and should be independent of the various information systems in use. The Crisis Standards of Care were chosen as basis and were operationalized in a seven-point Likert-scale for expert-based scoring: "No impact," "Buffer capacity needed," "Buffer capacity sufficient," "Unusual adaptations to care needed," "Unusual adaptations sufficient," "Disturbance of continuity of care inevitable without external assistance," and "Disturbance of continuity of care inevitable." Results: During the 2017 and 2018 winter/flu-seasons, crisis managers of ten hospitals scored the scale almost daily for three months. This served as a regional monitor and created the possibility to distribute patients and resources more evenly over the hospitals and with the care sector.

Discussion: The impact scale improved communication and mutual understanding between hospitals and with other health care organizations, and is expected to have helped in maintaining the continuity of care during the 2017 and 2018 winter/ flu-seasons. More research is needed on the reliability of the response. Nevertheless, the scale has since become an integral part of the regional contingency planning.

Prehosp Disaster Med 2019;34(Suppl. 1):s138

doi:10.1017/S1049023X19003054

Implementing Guidelines for Ambulance Services Dr. Rubije Hodza-Beganovic, Mr. Henrik C. Carlsson, Mr. Henrik Lidberg, Dr. Peter Berggren

KMC/IMP, Linköping, Sweden 